\title{
Inertial capability index based on fuzzy data
}

\author{
B.S. Gildeh ${ }^{\star}$ and S. Asghari ${ }^{\star \star}$ \\ Department of Statistics, Faculty of Mathematical Science, University of Mazandaran, 47417-95447 Babolsar, Iran
}

Received: 9 March 2011 / Accepted: 14 March 2011

\begin{abstract}
Process performance can be analyzed by using process capability indices (PCIs), which are summary statistics to depict the process location and dispersion successfully. In some cases, quality characteristic and target are not precise numbers and they are expressed in fuzzy terms, so that the classical capability indices cannot be applied. In this paper we obtain a confidence interval for inertial capability index $C_{p i}$ (defined by [Pillet, TQM Mag. 16, 202-209 (2004)]) based on fuzzy data and propose a membership function for it.
\end{abstract}

Keywords: Confidence interval; $D_{p, q \text {-distance }}$ fuzzy random variable; membership function; inertial tolerancing; inertial capability index

\section{Introduction}

Many PCIs have been proposed in the literature. Although they are very usable statistics to summarize process performance, they can give misleading results and can cause incorrect interpretation if the process distribution is not normal. Experience shows that the normality assumption is not often met in real world application and different distributions are presented such as skew, heavy-tailed and short tailed distributions and the percentages on nonconforming parts are significantly different from the computed PCIs.

The traditional tolerancing considers the conformity of a batch when it satisfies the specifications. The characteristic is considered for itself and not regarding its incidence on the final assembly resultant. The inertia $I=\sqrt{\sigma^{2}+\delta^{2}}$ is not tolerated by a tolerance interval but by a scalar representing the maximum inertia that the characteristic should not exceed. It has been showed that inertial tolerancing proposes another tolerancing method to guarantee the final assembly while allowing larger variability in the case of centered production. The inertial process capability index $\left(C_{p i}\right)$ is defined based on the inertial tolerancing that it has many properties, in particularly in the case of the mixed batches and it is independent from process distribution. One of the disadvantages of the traditional capability index such as $C_{p k}$ appears when two batches are mixed. By mixing two batches with an acceptable capability $\left(C_{p k}>1.33\right)$, one can obtain a non-acceptable capability $\left(C_{p k}<1.33\right)[1]$. These disadvantages are eliminated in the case of inertial tolerance with the $C_{p i}$ index [1].

^ Correspondence: sadeghpour@umz.ac.ir

${ }^{\star \star}$ Correspondence: Samane.asghari@yahoo.com
Chen et al. [2] used the fuzzy analytic method concerning process capability index $C_{p m}$ and calculated $\tilde{C}_{p m}$ for fuzzy observations. Perakis and Xekalaki [3] constructed confidence interval for the index $C_{p m}$ with crisp data. Parchami et al. [4] obtained fuzzy confidence interval for a fuzzy process capability index. In this paper, fuzzy set theory is incorporated to increase $C_{p i}$ 's flexibility and sensitivity by defining quality characteristic and target as fuzzy numbers. In such a case, both a confidence interval and a membership function for $C_{p i}$ are introduced. The fuzzy set theory is a useful method for modeling the problems with fuzzy (imprecise) information that has been recognized as one of uncertainties in the real world.

\section{Preliminary notes}

Definition 2.1. Let $R$ be the set of real numbers. Set, $F(R)=\{\tilde{A} \mid \tilde{A}: R \rightarrow[0,1], \tilde{A}$ is a continous function $\}$ and $F_{T}(R)=\{T(a, b, c) \mid a, b, c \in R, a \leqslant b \leqslant c\}$, where

$$
T(a, b, c)= \begin{cases}(x-a) /(b-a) & \text { if } a \leqslant x \leqslant b \\ (c-x) /(c-b) & \text { if } b \leqslant x \leqslant c \\ 0 & \text { elsewhere }\end{cases}
$$

Any $\tilde{A} \in F(R)$ is called a fuzzy set on $R$ and any $T(a, b, c) \in F_{T}(R)$ is called a triangular fuzzy number.

Definition 2.2. Fuzzy number $\tilde{A}$ is positive (negative) if

$$
\forall x \leqslant 0 ; \quad \tilde{A}(x)=0(\forall x \geqslant 0 ; \quad \tilde{A}(x)=0) .
$$

Definition 2.3. $a$-cut set for a fuzzy number is closed 
interval that indicated with $\left[A_{\alpha}^{-}, A_{\alpha}^{+}\right]$such that

$$
\begin{aligned}
& A_{\alpha}^{-}=\inf \{x \in R: \tilde{A}(x) \geqslant \alpha\}, \\
& A_{\alpha}^{+}=\sup \{x \in R: \tilde{A}(x) \geqslant \alpha\} .
\end{aligned}
$$

Definition 2.4. The $D_{p, q \text {-distance, indexed by parameters }}$ $1 \leqslant p \leqslant \infty$ and $0<q<1$ between two fuzzy numbers $\tilde{A}$ and $\tilde{B}$ is a nonnegative function on $F(R) \times F(R)$ gives as follows:

$$
D_{p, q}(\tilde{A}, \tilde{B})=\left\{\begin{array}{l}
{\left[(1-q) \int_{0}^{1}\left|A_{\alpha}^{-}-B_{\alpha}^{-}\right|^{p} d \alpha\right.} \\
\left.+q \int_{0}^{1}\left|A_{\alpha}^{+}-B_{\alpha}^{+}\right|^{p} d \alpha\right]^{\frac{1}{p}} \quad \text { if } p<\infty \\
(1-q) \sup _{0<\alpha \leqslant 1}\left(\left|A_{\alpha}^{-}-B_{\alpha}^{-}\right|\right) \\
+q \inf _{0<\alpha \leqslant 1}\left(\left|A_{\alpha}^{+}-B_{\alpha}^{+}\right|\right) \quad \text { if } p=\infty .
\end{array}\right.
$$

The analytical properties of $D_{p, q}$ depend on the first parameter $p$, while the second parameter $q$ of $D_{p, q}$ characterizes the subjective weight attributed to the sides of the fuzzy numbers. If there is no reason to distinguish any side of fuzzy numbers, $D_{p, 0.5}$ is recommended.

Definition 2.5. A mapping $\tilde{X}: \Omega \rightarrow F(R)$ is said to be a fuzzy random variable associated with $(\Omega, A)$ if and only if

$$
\left\{(\omega, x): x \in X_{\alpha}(\omega)\right\} \in \Omega \times B,
$$

where $B$ denote the $\sigma$-field of Borel set in $R$.

Definition 2.6. The central $D_{2, q}$-mean square dispersion of $\tilde{X}$ about $\tilde{E}(\tilde{X})\left(\right.$ or $\left.\tilde{\mu}_{\tilde{X}}\right)$ is called $D \operatorname{var}(\tilde{X})$ given by the value (if it exists)

$$
\begin{aligned}
& D \operatorname{var}(\tilde{X})=E\left(\left[D_{2, q}\left(\tilde{X}, \tilde{\mu}_{\tilde{X}}\right)\right]^{2}\right) \\
& =\int_{\Omega}\left[(1-q) \int_{0}^{1}\left(X_{\alpha}^{-}(w)-\left(\mu_{\tilde{X}}\right)_{\alpha}^{-}\right)^{2} d \alpha\right. \\
& \left.+q \int_{0}^{1}\left(X_{\alpha}^{+}(w)-\left(\mu_{\tilde{X}}\right)_{\alpha}^{+}\right)^{2} d \alpha\right] d p(w) .
\end{aligned}
$$

Proposition 1. Assume that $\tilde{A}$ and $\tilde{B}$ are triangular fuzzy numbers: $\tilde{A}=\operatorname{tri}\left(a_{1}, a_{2}, a_{3}\right)$ and $\tilde{B}=\operatorname{tri}\left(b_{1}, b_{2}, b_{3}\right)$, the $\alpha$-cuts of $\tilde{A}$ and $\tilde{B}$ are as follows

$$
\begin{aligned}
& A_{\alpha}=\left[(1-\alpha) a_{1}+a_{2} \alpha, a_{3} \alpha+(1-\alpha) a_{4}\right], \\
& B_{\alpha}=\left[(1-\alpha) b_{1}+b_{2} \alpha, b_{3} \alpha+(1-\alpha) b_{4}\right] .
\end{aligned}
$$

It can establish that

$$
\begin{aligned}
{\left[D_{2,1 / 2}(\tilde{A}, \tilde{B})\right]^{2} } & =\frac{1}{6}\left[\left(b_{1}-a_{1}\right)^{2}+2\left(b_{2}-a_{2}\right)^{2}+\left(b_{3}-a_{3}\right)^{2}\right. \\
& \left.+\left(b_{1}-a_{1}\right)\left(b_{2}-a_{2}\right)+\left(b_{3}-a_{3}\right)\left(b_{2}-a_{2}\right)\right] .
\end{aligned}
$$

Proposition 2. [5] Suppose that $\tilde{X}$ be a fuzzy random variable and $\tilde{T}$ be a fuzzy number. Then

$$
\begin{aligned}
& E\left[D_{2, q}(\tilde{X}, \tilde{T})\right]^{2}=E\left[D_{2, q}\left(\tilde{X}, \tilde{\mu}_{\tilde{X}}\right)\right]^{2} \\
& +\left[D_{2, q}\left(\tilde{\mu}_{\tilde{X}}, \tilde{T}\right)\right]^{2}=D \operatorname{var}(\tilde{X})+\left[D_{2, q}\left(\tilde{\mu}_{\tilde{X}}, \tilde{T}\right)\right]^{2}
\end{aligned}
$$

Similarly, it can establish that

$$
\begin{gathered}
\quad \frac{1}{n} \sum_{i=1}^{n}\left[D_{2, q}\left(\tilde{X}_{i}, \tilde{T}\right)\right]^{2}=\frac{1}{n} \sum_{i=1}^{n}\left[D_{2, q}(\tilde{X}, \tilde{\bar{X}})\right]^{2} \\
+\left[D_{2, q}(\tilde{\bar{X}}, \tilde{T})\right]=\hat{D} \operatorname{var}(\tilde{X})+\left[D_{2, q}(\tilde{\bar{X}}, \tilde{T})\right]^{2} .
\end{gathered}
$$

\section{The inertial acceptance criterion}

The aim of tolerancing is to determine an acceptation criterion on the components characteristics $x_{i}$ to guarantee the quality of the assembly resultant $Y$. In the case of a good design, when the $x$ characteristic is produced on the target, the quality is optimal. As $x$ gets an offset from the target, the function of the assembly will be more sensitive to the conditions of use and the environment, and can lead to a non-satisfaction of the customer. By using the Taguchi's financial loss function associated with an offcentering from the target $\left(L=K(X-T)^{2}\right)$, the Inertial tolerancing, in the case of a batch, is defined by Pillet [1] as follows

$$
I_{X}=E(X-T)^{2}=\sqrt{\sigma_{X}^{2}+\delta_{X}^{2}}
$$

Here $I_{X}$ represents the inertia of the $x$ characteristic, $\delta_{X}$ corresponds to the batch off-centering to its target and $\sigma_{X}$ is its standard deviation.

To qualify the capability of a process with the inertial tolerancing, Pillet [1] defines the $C_{p i}$ capability index as:

$$
C_{p i}=\frac{I_{\max }}{I_{B a t c h}}=\frac{I_{\max }}{\sqrt{\sigma_{X}^{2}+\delta_{X}^{2}}}
$$

which indicates the capability considering the process offcentering.

Compared to the traditional tolerancing, the proposed approach of the inertial tolerancing is quite different. The aim is no more to guarantee a rate of parts out of tolerance, but to guarantee the centering of components around the target in order to guarantee the quality of the assembly. The reflection is no more based on the proportions out of tolerances but on the inertias of the components, the normality of the batch distribution is no more a necessary criterion [1]. The main advantage of the inertial acceptance criterion is the absence of use of a tolerance interval that usually corresponds to the acceptance limits of the parts dimension. There is then no ambiguity of acceptance of a batch included in the tolerance interval but not acceptable due to the $C_{p m}$ capability index [6].

\section{The inertial tolerancing method}

Inertial tolerancing is a statistical method of tolerance synthesis. The allocation strategy is similar to that of the traditional statistical tolerancing. Let us consider components with centered batches. The resultant assembly is 
then a centered batch. The functional requirement is defined by a tolerance interval that is supposed to contain six standard deviations of the resultant batch. As the component inertias in the case of centering are given by the batch standard deviations, the inertial tolerances of components under the assumption of independent variables are given by:

$$
I_{i}=\frac{R_{0 x}}{6 \sqrt{n}}
$$

where

$I_{i}=$ the inertial tolerance of the $i$ th components

$R_{0}=$ the tolerance interval of the functional requirement

$n=$ the number of components in the dimensions chain.

In the general case, the inertial tolerance allocation is given by:

$$
I_{i}=\frac{\beta_{i} R_{0 x}}{6 \sqrt{\alpha_{i}^{2} \beta_{i}^{2}}}
$$

where $\alpha_{i}$ corresponds to influential coefficients of the $i$ th component on the resultant assembly and $\beta_{i}$ is the feasibility index of the $i$ th component that allows a nonuniform distribution of the tolerances [6].

\section{The inertial acceptance criterion for fuzzy data}

Assume that $\tilde{Y}$ (fuzzy random variable) represents quality characteristic and $\tilde{T}$ (fuzzy number) represents target value. In this case we introduce the inertial for $\tilde{Y}$ and inertial capability index $C_{p i}$ based on the definitions given in Section 2 and Proposition 2 such as following

$$
\begin{gathered}
I_{\tilde{Y}}=E\left(D_{2, q}(\tilde{Y}, \tilde{\tau})\right)^{2}=D \operatorname{var}(\tilde{Y})+\left(D_{2, q}\left(\tilde{\mu}_{\tilde{Y}}, \tilde{T}\right)\right)^{2}, \\
C_{p i}=\frac{I_{Y \max }}{I_{\tilde{Y}}}=\frac{I_{Y \max }}{\sqrt{E\left(D_{2, q}(\tilde{Y}, \tilde{T})\right)^{2}}} \\
=\frac{I_{Y \max }}{\sqrt{D \operatorname{var}(\tilde{Y})+\left(D_{2, q}\left(\tilde{\mu}_{\tilde{Y}}, \tilde{T}\right)\right)^{2}}},
\end{gathered}
$$

and we estimate $\hat{C}_{p i}$ with the following relation

$$
\hat{C}_{p i}=\frac{I_{Y \max }}{\sqrt{\frac{1}{n} \sum_{j=1}^{n}\left(D_{2, q}\left(\tilde{Y}_{j}, \tilde{T}\right)\right)^{2}}} .
$$

\section{The interval estimation of $C_{p i}$ based on fuzzy data}

The statistic $\frac{\sum_{i=1}^{n}\left[D_{2,1 / 2}\left(\tilde{Y}_{i}, \tilde{T}\right)\right]^{2}}{D \operatorname{var}(\tilde{Y})}$, is distributed as the noncentral chi-square with $n$ degrees of freedom and noncentrality parameter $n \delta$ where $\delta=\frac{\left[D_{2,1 / 2}\left(\tilde{\mu}_{\tilde{Y}}, \tilde{T}\right)\right]^{2}}{D \operatorname{var}(\tilde{Y})},[5,7]$.

Therefore, it follows that

$$
\begin{aligned}
& P\left(\chi_{n, \alpha / 2}^{2}(n \delta)<\frac{\sum_{i=1}^{n}\left[D_{2,1 / 2}\left(\tilde{Y}_{i}, \tilde{T}\right)\right]^{2}}{D \operatorname{var}(\tilde{Y})}<\chi_{n, 1-\alpha / 2}^{2}(n \delta)\right) \\
& =1-\alpha \text {, }
\end{aligned}
$$

where $\chi_{n, \alpha / 2}^{2}(n \delta)$ denotes the $100 \alpha \%$ percentile of the noncentral chi-square distribution with $n$ degrees of freedom and non-centrality parameter $n \delta$. We can write

$$
\begin{gathered}
P\left(\chi_{n, \alpha / 2}^{2}(n \delta)<\frac{\frac{1}{n} \sum_{i=1}^{n}\left[D_{2, q}\left(\tilde{Y}_{i}, \tilde{T}\right)\right]^{2}}{E\left[D_{2, q}(\tilde{Y}, \tilde{T})\right]^{2}}\right. \\
\left.\times \frac{n E\left[D_{2, q}(\tilde{Y}, \tilde{T})\right]^{2}}{D \operatorname{var}(\tilde{Y})}<\chi_{n, 1-\alpha / 2}^{2}(n \delta)\right)=1-\alpha .
\end{gathered}
$$

Taking into account the fact that

$$
\left(\frac{C_{p i}}{\hat{C}_{p i}}\right)^{2}=\frac{\sum_{i=1}^{n}\left[D_{2, q}\left(\tilde{Y}_{i}, \tilde{T}\right)\right]^{2}}{n E\left[D_{2, q}(\tilde{Y}, \tilde{T})\right]^{2}},
$$

we obtained

$$
\begin{aligned}
& P\left(\frac{\chi_{n, \alpha / 2}^{2}(n \delta) D \operatorname{var}(\tilde{Y})}{n E\left[D_{2, q}(\tilde{Y}, \tilde{T})\right]^{2}}<\left(\frac{C_{p i}}{\hat{C}_{p i}}\right)^{2}\right. \\
& \left.<\frac{\chi_{n, 1-\alpha / 2}^{2}(n \delta) D \operatorname{var}(\tilde{Y})}{n E\left[D_{2, q}(\tilde{Y}, \tilde{T})\right]^{2}}\right)=1-\alpha .
\end{aligned}
$$

Let $\hat{\delta}=\frac{\left[\hat{D}_{2,1 / 2}\left(\tilde{\mu}_{\tilde{\tilde{Y}}}, \tilde{T}\right)\right]^{2}}{\hat{D} \operatorname{var}(\tilde{Y})}$, then the interval

$$
\left(\hat{C}_{p i} \sqrt{\frac{\chi_{n, \alpha / 2}^{2}(n \delta)}{n(1+\hat{\delta})}}, \hat{C}_{p i} \sqrt{\frac{\chi_{n, 1-\alpha / 2}^{2}(n \delta)}{n(1+\hat{\delta})}}\right)
$$

is a $100(1-\alpha) \%$ confidence interval for $C_{p i}$.

In order to evaluate the non-central chi-square distribution with $n$ degrees of freedom and non-centrality parameter $n \delta$ can use the order "ncx2inv" in MATLAB program or estimates with Patnaik's approximation [3].

To obtain a better understanding of $C_{p i}$ based on fuzzy data, we propose to put this confidence interval as a $\alpha$-cut for $C_{p i}$ and plot the upper and lower limits of the interval estimation for different values of $\alpha$. This method gives a membership function for $\tilde{C}_{p i}$. 
Table 1. Triangular fuzzy data collected from suppliers.

\begin{tabular}{|c|c|c|c|}
\hline$\underline{S_{1}}$ & $S_{2}$ & $S_{3}$ & $S_{4}$ \\
\hline$(68,26,70,46,72.18)$ & $(72,69,73,96,75.20)$ & $(54,24,64,27,70,69)$ & $(58.53,60.58,66.15)$ \\
\hline$(70.22,72.88,73.10)$ & $(57.90,58.69,60.26)$ & $(66.67,72.00,73.61)$ & $(54.79,61.16,69.86)$ \\
\hline$(62,26,63.52,66.82)$ & $(76,09,77,08,79.03)$ & $(66,31,70,38,74,15)$ & $(58.37,58.92,72.53)$ \\
\hline$(66.64,68.10,70.09)$ & $(64.40,66.80,68.54)$ & $(59.89,66.92,71.78)$ & $(55.20,56.13,66.92)$ \\
\hline$(67,80,69,17,70.22)$ & $(67,28,68,07,69,70)$ & $(69,28,70,23,73,45)$ & $(59.92,65.08,65.57)$ \\
\hline$(65.33,66.79,68.20)$ & $(64.46,65.61,67.98)$ & $(65.90,66.92,67.90)$ & $(65.16,70.91,73.60)$ \\
\hline$(59.90,61.71,62.90)$ & $(65.90,67.48,68.67)$ & $(62.73,63.03,64.02)$ & $(54.03,58.64,61.83)$ \\
\hline$(68.54,69.38,70.32)$ & $(67.67,68.12,69.29)$ & $(59.88,60.12,62.54)$ & $(66.50,66.63,72.08)$ \\
\hline$(72,10,73,28,74,90)$ & $(67,25,68,62,68,98)$ & $(69,25,70.03,71,32)$ & $(62.17,65.77,66.84)$ \\
\hline$(72,19,74,26,75,32)$ & $(60.80,61.01,62.20)$ & $(70,00,71,39,72.12)$ & $(65,24,73,35,79,83)$ \\
\hline$(72.69,73.96,75.20)$ & $(65.90,66.92,67.90)$ & $(63.89,64.74,66.23)$ & $(65.65,70.76,72.17)$ \\
\hline$(57.90,58.69,60.26)$ & $(62,73,63,03,64,02)$ & $(65.80,66.85,67.92)$ & $(68,26,72,26,75,81)$ \\
\hline$(76.09,77.08,79.03)$ & $(59.88,60.12,62.54)$ & $(54.42,55.75,57.34)$ & $(63.88,64.98,71.10)$ \\
\hline$(64,40,66.80,68.54)$ & $(69,25,70.03,71,32)$ & $(67,56,69,47,70,22)$ & $(61,98,62.58,74,81)$ \\
\hline$(67.28,68.07,69.70)$ & $(70,00,71.39,72.12)$ & $(59.42,60.12,61.78)$ & $(58.42,59.82,63.24)$ \\
\hline$(64,46,65,61,67.98)$ & $(63.89,64.74,66.23)$ & $(64,90,68,48,69.67)$ & $(58.59,68.87,70.05)$ \\
\hline$(65.90,67.48,68.67)$ & $(65.80,66.85,67.92)$ & $(62,69,63.22,65.79)$ & $(55.25,66.51,68.72)$ \\
\hline$(67.67,68,12,69,29)$ & $(54,42,55,75,57,34)$ & $(68,35,69,72,70,98)$ & $(54.72,66.88,74,94)$ \\
\hline$(67,25,68,62,68.98)$ & $(67,56,69,47,70,22)$ & $(62,94,63.51,64.20)$ & $(63.69,65.03,75.51)$ \\
\hline$(60.80,61.01,62.20)$ & $(59.42,60.12,61.78)$ & $(65.30,66.96,67.65)$ & $(57.63,61.54,66.43)$ \\
\hline
\end{tabular}

\section{A numerical example}

In this section, we use Example 6.1 given in [8], that is described in the following.

Since light emitting diodes (LEDs) have a long life span and high intensity of solid-state illumination exhibiting a wide range of colors, the uses of LEDs are growing rapidly in a wide variety of applications such as automotive lighting, computer displays, LCD televisions, signaling and general lighting products.

Here, an LED-based lighting fixture (LED-LF) is investigated as an example; the LED-LF is manufactured in Tainan Industrial Park, Taiwan. Due to high demands on the LED-LFs, the company does not have enough production capacity to supply one type of LED components used in the LED-LFs. Therefore, the decision-makers decide to purchase the LED components from some possible suppliers. The luminous intensity of LED sources is a critical characteristic for this type of LEDs. Thus far, all light measurements and rating systems depend on the perception of the human eye or imprecise terminology and calibration standards. This implies that the randomness is not the only aspect of uncertainty for data collected on the luminous intensity of LED sources; that is, the occurrence of fuzziness introduces another uncertainty that should be taken into account while solving the problem. Four suppliers are capable of producing this type of LEDs. The decision-makers need to choose preferable suppliers based on the fuzzy sample data of the luminous intensity which have been collected from each supplier with size 20, as listed in Table 1, where the data $\tilde{x}_{i n}=\operatorname{tri}\left(x_{i n 1}, x_{i n 2}, x_{i n 3}\right)$ with $i=1,2,3,4$ and $n=1,2$, $3, \ldots 20$, are assumed as triangular fuzzy numbers. The upper and lower specification limits of luminous intensity are set at $U S L=90 \mathrm{mcd} / \mathrm{m}^{2}$ and $L S L=40 \mathrm{mcd} / \mathrm{m}^{2}$, respectively.

Let the value of target be equal to total mean of fuzzy data, that is $\tilde{T}=\operatorname{tri}(64.1046,66.3670,68.9561)$.
Necessary maximum inertial with method in Section 4 is obtained such as $I_{\max }=8.3333$.

We obtained the value of $C_{p i}$ for each supplier such as following:

\begin{tabular}{cc}
\hline Supplier & $C_{p i}$ \\
\hline $\mathrm{S}_{1}$ & 1.6976 \\
$\mathrm{~S}_{2}$ & 1.6307 \\
$\mathrm{~S}_{3}$ & 1.9645 \\
$\mathrm{~S}_{4}$ & 1.7412 \\
\hline
\end{tabular}

According to $C_{p i}$, the order of four suppliers is ranked as $\left\{\mathrm{S}_{3}, \mathrm{~S}_{4}, \mathrm{~S}_{1}, \mathrm{~S}_{2}\right\}$.

In order to obtain a membership function for $C_{p i}$, we construct confidence intervals against different values of $\alpha$. Figure 1 shows the graph of $\tilde{C}_{p i}$ for each supplier. The membership functions of $\tilde{C}_{p i}$ 's are triangular fuzzy numbers, given as follows:

\begin{tabular}{cc}
\hline Supplier & $\tilde{C}_{p i}$ \\
\hline $\mathrm{S}_{1}$ & $T(0.8853,1.6700,2.6044)$ \\
$\mathrm{S}_{2}$ & $T(0.8472,1.6034,2.5127)$ \\
$\mathrm{S}_{3}$ & $T(1.0206,1.9316,3.0273)$ \\
$\mathrm{S}_{4}$ & $T(0.9090,1.7132,2.6681)$ \\
\hline
\end{tabular}

For comparison $\tilde{C}_{p i}$ 's, one can calculate their $D_{p, q \text {-distance }}$ from zero.

\begin{tabular}{cl}
\hline Supplier & $D_{p, q}$ \\
\hline $\mathrm{S}_{1}$ & 3.1620 \\
$\mathrm{~S}_{2}$ & 2.9268 \\
$\mathrm{~S}_{3}$ & 4.2480 \\
$\mathrm{~S}_{4}$ & 3.3239 \\
\hline
\end{tabular}

According to this method of ranking, four suppliers are ranked as $\left\{\mathrm{S}_{3}, \mathrm{~S}_{4}, \mathrm{~S}_{1}, \mathrm{~S}_{2}\right\}$. 


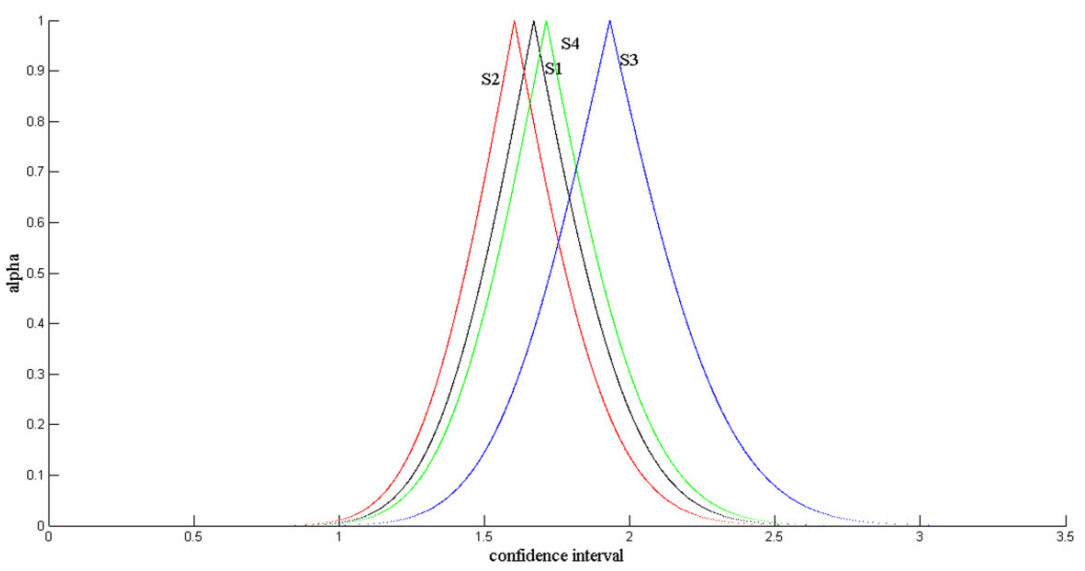

Fig. 1. Membership function of $C_{p i}$ for each supplier.

\section{Conclusion}

A constructive methodology for obtaining the fuzzy estimate of inertial process capability index $C_{p i}$ with the help of "confidence interval" and " $D_{p, q \text {-distance" is proposed in }}$ this paper. The main advantage of this methodology is that the fuzzy data can be handled now. By applying the $D_{p, q \text {-distance }}$ between two fuzzy numbers, we estimated $\tilde{C}_{p i}$ and proposed a method to compare different $\tilde{C}_{p i}$ 's. Our results are illustrated by a numerical example.

Acknowledgements. The authors would like to express their sincere thanks to the referees for their valuable comments and suggestions.

\section{References}

1. M. Pillet, Inertial tolerancing, TQM Mag. 16, 202-209 (2004)

2. C.C. Chen, C.M. Lai, H.Y. Nien, Measuring process capability index $C p m$ with fuzzy data, Qual. Quant. 44, 529535 (2010)
3. P.B. Patnaik, The non-central $\chi^{2}$ and F-distributions and their applications, Biometrika 36, 202-232 (1949)

4. A. Parchami, M. Mashinchi, H.R. Maleki, Fuzzy confidence interval for fuzzy process capability index, J. Intell. \& Fuzzy Syst. 17, 287-295 (2006)

5. B.S. Gildeh, D. Gien, $D_{p, q \text {-distance }}$ and the correlation coeficient between two fuzzy random variables, Rencontres francophones sur la logique floue et ses applications (Mons, Belgique 2001), pp. 97-101

6. P.A. Adragna, S. Samper, M. Pillet, A proposition of 3D inertial tolerancing to consider the statistical combination of the location and orientation deviations, Int. J. Product Development, IJPD 10, 26-45 (2010)

7. N.N. Vakhania, Probability distribution on linear space (Elsevier science publishes, B.V. North holland, 1981)

8. M.H. Shu, H.C. Wu, Quality-based supplier selection and evaluation using fuzzy data, Comput. Ind. Eng. 57, 10721079 (2009)

9. P.A. Adragna, M. Pillet, F. Formosa, S. Samper, Inertial tolerancing and capability indices in an assembly production, Revue Internationale d'Ingénierie Numérique 2, 7188 (2006)

10. M. Perakis, E. Xekalaki, A new method for constructing Confidence intervals for the index Cpm, Qual. Reab. Eng. Int. 20, 651-665 (2004) 